\title{
Linguistic tuple segmentation in ngram-based statistical machine translation
}

\author{
Adrià de Gispert, José B. Mariño \\ TALP Research Center \\ Universitat Politècnica de Catalunya (UPC), Barcelona \\ \{agispert|canton\}@gps.tsc.upc.edu
}

\begin{abstract}
Ngram-based Statistical Machine Translation relies on a standard Ngram language model of tuples to estimate the translation process. In training, this translation model requires a segmentation of each parallel sentence, which involves taking a hard decision on tuple segmentation when a word is not linked during word alignment. This is especially critical when this word appears in the target language, as this hard decision is compulsory.

In this paper we present a thorough study of this situation, comparing for the first time each of the proposed techniques in two independent tasks, namely English-Spanish European Parliament Proceedings large-vocabulary task and Arabic-English Basic Travel Expressions small-data task. In the face of this comparison, we present a novel segmentation technique which incorporates linguistic information. Results obtained in both tasks outperform all previous techniques.
\end{abstract}

Index Terms: statistical machine translation, tuple segmentation, n-gram-based SMT, linguistic information

\section{Introduction}

Ngram-based (or Tuple-based) Statistical Machine Translation (SMT) has proved to be a state-of-the-art alternative approach to phrase-based models in performance comparisons [1,2]. Its main distinctive treat is the estimation of the core translation model by means of a standard Ngram language model, defined on the special bilingual language expressed by tuples [3].

According to literature, tuples are units containing one or more source-language words and one or more target-language words (including the NULL token, which is in fact no word). This model has its origins in machine translation using Finite-State Transducers [4], whose theoretical foundation expresses that we seek the target sentence 'e' maximising:

$$
\prod_{n=1}^{N} p\left((f, e)_{n} \mid(f, e)_{n-x+1}, \ldots,(f, e)_{n-1}\right)
$$

where the $\mathrm{n}$-th tuple of a sentence pair is referred as $(f, e)_{n}$. In order to estimate this language model parameters, given a parallel corpus and a certain word alignment, and in contrast to phrasebased approaches, the training requires a unique segmentation of each bilingual pair of sentences into a sequence of tuples so that the natural order of both languages is not violated [5].

The standard tuple extraction algorithm [5] defines a unique set of tuples except whenever the resulting tuple contains no source word (NULL-source tuple). In order to re-use these units in decoding new sentences, the search should allow for no input word to generate units, and this is not the case. Therefore, these units cannot be allowed, and a certain hard decision must be taken regarding tuple segmentation, as in figure 1.

Literature offers examples of criteria to decide this segmentation, ranging from simply linking the target words of the NULLsource tuple to the previous or next tuple (if there is any) deterministically, to comparing the IBM model 1 score assigned to the resulting tuples of two competing segmentations [6].

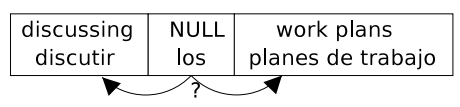

Figure 1: A hard segmentation decision must be taken

However, to our knowledge the impact of this segmentation decision on translation quality is not studied, nor the alternative segmentation strategies are compared. Here, we carry out this comparison, exploring the impact of this hard decision in Ngram translation model estimation and in translation quality, and we propose a novel strategy which follows a linguistic criteria to indirectly reduce the model entropy. We perform this comparison in an English-to-Spanish (and viceversa) large-vocabulary task, and additionally in an Arabic-to-English small-vocabulary task. Furthermore, we study the effect on translation of NULL tokens when these occur in the target side of the tuple.

\section{Tuple Segmentation strategies}

According to the aforementioned conceptual framework of Ngram translation model, it seems clear that the ideal tuple segmentation strategy should take a global decision based on the segmentation for all other NULL-source cases, attempting to obtain that set of tuples and Ngrams which better represented the unseen universe of events, meaning the one with less entropy. However, no feasible algorithm can perform that calculation in a reasonable time given current computational power, as this would involve a whole model reestimation for each particular segmentation alternative.

So far two segmentation strategies to solve the source NULL problem have been proposed, which are presented in the next sections, together with a novel strategy based on a linguistic criterion.

\subsection{Deterministic always NEXT}

A very pragmatic and simple approach is to always join the target words involved in NULL links (NULL-linked words) to the next tuple, if there is any (otherwise to the previous one), as first done in [7]. Besides simplicity and extreme efficiency, we do not observe any other advantage of this approach, which on the other hand does not follow any linguistic or statistical criterion. 


\subsection{IBM model 1 weight}

Being independent of word position, IBM model 1 probabilities provide a probabilistic lexicon between pairs of word of each language (see [8] for details in these models). This information can be used to weight and compare the resulting tuples from two competing segmentations, is in [6].

While this approach is appealing in that it uses bilingual information, observation of these situations leads to a different conclusion; many NULL-linked words represent articles, prepositions, conjunctions and other particles whose main function is to ensure the grammatical correctness of a sentence, complementing other more informative words. Therefore, their probabilities to translate to another word are not very meaningful.

\subsection{Entropy of the POS distribution}

Alternatively, from a linguistic point of view, one can think of this tuple segmentation problem around source NULLs as a monolingual decision related to whether the given target word is more connected to the preceding or to the following word. Intuitively, we can expect that a good criterion to perform tuple segmentation lays in preserving grammatically-connected phrases (such as, for instance, articles together with the noun they precede) in the same tuple, as this may probably lead to a simplification of the translation task. On the contrary, splitting linguistic units into separate tuples will probably lead to a tuple vocabulary increase and a higher sparseness, producing a poorer Ngram model.

In this direction, we propose to take the segmentation decision according to the entropy of the forward and backward Part-OfSpeech (POS) distributions, which we define conditioned to context. In detail, given the tuple sequence described as follows:

$$
\begin{array}{ccc}
<\ldots s_{j}> & \text { NULL } & <s_{j+1} \ldots> \\
\mid & \mid & \mid \\
<\ldots t_{i-1}> & t_{i} & <t_{i+1} \ldots>
\end{array}
$$

where $s_{j}$ means word in position $j$ in source sentence, and equivalently $t_{i}$ means word in position $i$ in target sentence, we can define a 'forward' entropy of the POS distribution in position $i+1$ given $\left(t_{i-1}, t_{i}\right)$ as in equation 2 :

$$
H_{P O S}^{f}=-\sum_{P O S} p_{P O S}^{f} \log p_{P O S}^{f}
$$

where

$$
p_{P O S}^{f}=\frac{N\left(t_{i-1}, t_{i}, P O S_{i+1}\right)}{\sum_{P O S^{\prime}} N\left(t_{i-1}, t_{i}, P O S_{i+1}^{\prime}\right)}
$$

is the probability of observing a certain Part-Of-Speech following the sequence of words defined by $t_{i}$ and $t_{i+1}$.

Equivalently, we can define a 'backward' entropy of the POS distribution in position $i-1$ given $\left(t_{i}, t_{i+1}\right)$ as in equation 4 :

$$
H_{P O S}^{b}=-\sum_{P O S} p_{P O S}^{b} \log p_{P O S}^{b}
$$

where

$$
p_{P O S}^{b}=\frac{N\left(P O S_{i-1}, t_{i}, t_{i+1}\right)}{\sum_{P O S^{\prime}} N\left(P O S_{i-1}^{\prime}, t_{i}, t_{i+1}\right)}
$$

is the probability of observing a certain Part-Of-Speech preceding the sequence of words defined by $t_{i-1}$ and $t_{i}$.
Then, we can take a tuple segmentation decision favouring the most POS-entropic case. The rationale behind this is that, if $H_{P O S}^{f}>H_{P O S}^{b}$, we have observed the first sequence of words comprised of $\left(t_{i-1}, t_{i}\right)$ in more grammatically different situations than the latter sequence comprised of $\left(t_{i}, t_{i+1}\right)$. Therefore, we can induce that $t_{i-1}$ and $t_{i}$ tend to be more often connected than $t_{i}$ and $t_{i+1}$, and should belong to the same translation tuple. Analogously, one can conclude the contrary if $H_{P O S}^{f}<H_{P O S}^{b}$.

While this is a monolingual decision on the target language morphology, being linguistically-guided, the POS entropy approach is much more correlated with human intuition.

\subsection{Criteria for NULLs in target}

Whereas the segmentation decision is required when a target word is unlinked (or linked to NULL), this is not so when the unlinked word is in the source target, in which case these units are allowed in the tuple vocabulary for Ngram estimation .

However, one can think of applying the same criterion to remove NULLs in the target side of tuples, possibly addressing omission errors in translation. Aiming at evaluating the impact of this decision, we have also applied the POS entropy strategy to segment tuples with unlinked source words.

\section{Experimental framework}

In order to compare each segmentation strategy and evaluate its impact on translation quality, experiments have been carried out using two parallel corpora, differing in language pair and corpus size. On the one hand, we used a Spanish-English largevocabulary corpus, containing the European Parliament Proceed-

\begin{tabular}{|c|c|c|c|c|c|}
\hline & sent & words & vocab & avglen & refs \\
\hline \multicolumn{6}{|l|}{ Train set } \\
\hline English & \multirow{2}{*}{$1.22 \mathrm{M}$} & $33.37 \mathrm{M}$ & $104.8 \mathrm{k}$ & 27.3 & \multirow{2}{*}{1} \\
\hline Spanish & & $34.96 \mathrm{M}$ & $151.4 \mathrm{k}$ & 28.6 & \\
\hline \multicolumn{3}{|l|}{ Dev set } & \multicolumn{3}{|c|}{ OOVs } \\
\hline English & \multirow{2}{*}{504} & $15.3 \mathrm{k}$ & $2.30 \mathrm{k}$ & 20 & 3 \\
\hline Spanish & & $15.4 \mathrm{k}$ & $2.74 \mathrm{k}$ & 22 & 3 \\
\hline \multicolumn{3}{|l|}{ Test set } & \multicolumn{3}{|c|}{ OOVs } \\
\hline English & 840 & $22.75 \mathrm{k}$ & $4.1 \mathrm{k}$ & 44 & 2 \\
\hline Spanish & 1094 & $26.88 \mathrm{k}$ & $4.0 \mathrm{k}$ & 113 & 2 \\
\hline
\end{tabular}
ings from 1996 to September 2004, and on the other, an Arabicto-English small-vocabulary corpus, which contains a small part of the Basic Travel Expressions Corpus. English has been tagged using TnT tagger ${ }^{1}$, and Spanish using FreeLing analysis tool ${ }^{2}$.

Table 1: European Parliament English-Spanish corpus statistics.

Statistics of each corpus can be found in Tables 1 and 2, including number of sentences, running words, vocabulary sizes, average sentence length and out-of-vocabulary (OOVs) words and number of reference translations for development and test sets. Note the big size difference between tasks.

\subsection{Translation model results}

A comparison of Ngram translation model performance for each task is shown in rows named 'alwaysNEXT', 'IBM1weight' and

\footnotetext{
${ }^{1}$ Available at www.coli.uni-saarland.de/ thorsten/tnt

${ }^{2}$ Available at http://garraf.epsevg.upc.es/freeling
} 


\begin{tabular}{|c|c|c|c|c|c|}
\hline & sent & words & vocab & avglen & refs \\
\hline \multicolumn{6}{|l|}{ Train set } \\
\hline Arabic & 201 & $180.5 \mathrm{k}$ & $16.0 \mathrm{k}$ & 9.0 & 1 \\
\hline English & $20 \mathrm{~K}$ & $189.2 \mathrm{k}$ & $7.2 \mathrm{k}$ & 9.5 & 1 \\
\hline \multicolumn{3}{|l|}{ Dev set } & \multicolumn{3}{|c|}{ OOVs } \\
\hline Arabic & 506 & $3.63 \mathrm{k}$ & $1.18 \mathrm{k}$ & 196 & 16 \\
\hline \multicolumn{3}{|l|}{ Test set } & \multicolumn{3}{|c|}{ OOVs } \\
\hline Arabic & 1006 & $7.22 \mathrm{k}$ & $1.9 \mathrm{k}$ & 356 & 16 \\
\hline
\end{tabular}

Table 2: Travel Expressions Arabic-English corpus statistics.

\begin{tabular}{|c|c|c|c|c|}
\hline \multicolumn{2}{|c|}{} & BLEU & mWER & NIST \\
\hline \hline \multirow{5}{*}{$\mathrm{E} \rightarrow \mathrm{S}$} & alwaysNEXT & 0.4215 & 43.98 & 9.22 \\
& IBM1weight & 0.4221 & 43.60 & 9.19 \\
& POSentropy & $\mathbf{0 . 4 3 2 5}$ & $\mathbf{4 3 . 4 8}$ & $\mathbf{9 . 3 0}$ \\
\cline { 2 - 5 } & trgNULL & 0.4249 & 44.47 & 9.21 \\
& trgNULLpos & 0.4313 & 43.75 & 9.29 \\
\hline \hline \multirow{5}{*}{$\mathrm{S} \rightarrow \mathrm{E}$} & alwaysNEXT & 0.4661 & 39.37 & 9.86 \\
& IBM1weight & 0.4698 & 38.73 & 9.91 \\
& POSentropy & $\mathbf{0 . 4 7 5 6}$ & $\mathbf{3 8 . 6 4}$ & $\mathbf{9 . 9 5}$ \\
\cline { 2 - 5 } & trgNULL & 0.4728 & 39.23 & 9.91 \\
& trgNULLpos & 0.4733 & 38.78 & 9.93 \\
\hline \hline \multirow{3}{*}{$\mathrm{A} \rightarrow \mathrm{E}$} & alwaysNEXT & 0.3684 & 41.80 & 7.16 \\
& IBM1weight & 0.3656 & 41.94 & 7.14 \\
& POSentropy & $\mathbf{0 . 3 6 9 1}$ & 41.91 & $\mathbf{7 . 1 7}$ \\
\hline
\end{tabular}

Table 3: Translation model performance for each segmentation strategy. 'E' stands for English, 'S' for Spanish and 'A' for Arabic.

'POSentropy' in Table 3, refering to each segmentation strategy discussed in sections 2.1 to 2.3 , respectively.

Regarding the large-vocabulary tasks, the proposed linguistically-guided segmentation outperforms all other strategies significantly, especially in the Eng $\rightarrow$ Spa direction. This result is consistent with the fact that Spanish is a more word-generative language than English, and therefore, more NULLs are found in the English side of extracted tuples.

Even though the impact of changing the segmentation criterion when translating into English is smaller, the improvement of the POSentropy approach is significant. In the small-vocabulary Ara $\rightarrow$ Eng task differences are less significant, in correlation with the fact that only $7 \%$ of tuples contain NULLs in Arabic side, compared to the $14 \%$ of Eng $\rightarrow$ Spa task.

Remarkably, whereas IBM1weight provides better results in large-vocabulary tasks than the alwaysNEXT criterion, the result is opposite in the small-vocabulary Ara $\rightarrow$ Eng task. On the other hand, the POSentropy approach proves to be more general and robust to a task change, achieving best performance in all tasks.

\subsection{Removing NULLs in target}

Following the idea suggested in section 2.4, Table 3 also presents results when applying the best segmentation criterion (POSentropy) to avoid NULLs in the tuples target side, as shown in rows named 'trgNULL' and 'trgNULLpos' for large-vocabulary Spanish-English task. The first refers to applying the criterion to all tuples, whereas the latter to only applying it when the tuple contains a POS of a Noun, Adjective or Verb. The objective of this is to minimise omission errors by preventing tuples with content words in source side and NULL in target to belong to the model dictionary.

However, results show that none of these techniques is beneficial. Clearly, and in contrast to NULLs in the tuple source side, NULLs in the target side are a useful mechanism for the Ngram model to find good contexts and significantly increase performance regardless of the translation direction. This holds even when we allow tuples with content words in source side and NULL in target.

\subsection{Translation Ngrams study}

To better understand these results, Table 4 shows the tuple vocabulary obtained in training for each segmentation (tup vcb), and relevant statistics of translated output, namely the percentage of tuples used as 1-grams, 2-grams and 3-grams during translation, the average tuple length obtained (measuring source and target sides separately) and the number of tuples with NULL in target (in the translated output).

Regarding tuple vocabulary size, the alwaysNEXT criterion produces the biggest vocabulary in training when compared to POSentropy and IBM1weight, which produces the smallest. When removing the target NULLs, the vocabulary size is significantly improved.

In Eng $\rightarrow$ Spa, we observe that translation with alwaysNEXT and POSentropy segmentation criteria tend to use more 3 grams than IBM1weight, which can be explained by their consistency in taking segmentation decisions (they invariably take the same decision given the target words involved), whereas IBM1weight depends on source and target words and is more variable.

However, using more 3 grams is not directly correlated with translation scores, and the number of tuples to NULL needs to be taken into account. The high number of tuples to NULL for the alwaysNEXT criterion is outstanding, and tells us that translation is indeed achieving many 3 grams by catenating sequences to NULL, which do not necessarily achieve better performance. In the case of IBM1weight and especially of POSentropy, the number of tuples with NULL in target is strongly reduced. Whereas this appears to be positive for translation performance, when completely or partially removing NULLs in target (trgNULL and trgNULLpos), average tuple length increases, not only in the source side but also in the target side, and the model looses tuple context and falls much more often to 1 gram. Apparently, this has a negative effect in translation quality.

Therefore, we can conclude that the best relationship between high-order tuple context and small amount of tuples to NULL is achieved by the proposed POSentropy segmentation criterion. Differences are much smaller in the $\mathrm{Spa} \rightarrow$ Eng direction, although the same tendency in number of tuples with target NULL is to be found, and conclusions are analogous.

\subsection{Translation model + features results}

To further evaluate the impact of these segmentation differences, we have log-linearly combined the Ngram translation model with additional features, namely two lexicon features based on IBM model 1 probabilities, a standard target 3-gram Language Model and a constant word bonus, and optimized according to BLEU score in development set, as similarly done in [3]. Table 5 shows the translation results for the two best segmentations for each task. As it can be seen, the improvement of better segmenting tuples with NULLs in source is practically compensated by the contribution of additional features, especially in the $\mathrm{Spa} \rightarrow$ Eng task. 


\begin{tabular}{|c|c|c|c|c|c|c|c|c|}
\hline & \multicolumn{4}{|c|}{ Eng $\rightarrow$ Spa } & \multicolumn{4}{|c|}{ Spa $\rightarrow$ Eng } \\
\hline & tup vcb & \% 1-2-3grams & tup len & null & tup vcb & $\%$ 1-2-3grams & tup len & null \\
\hline alwaysNEXT & $2.11 \mathrm{M}$ & $17.6-44.4-38.0$ & $1.16-1.10$ & 3119 & $2.15 \mathrm{M}$ & $14.1-41.5-44.4$ & $1.14-1.06$ & 2761 \\
\hline IBM1weight & $2.04 \mathrm{M}$ & $18.0-44.7-37.3$ & $1.16-1.09$ & 2466 & $2.08 \mathrm{M}$ & $14.2-41.4-44.4$ & $1.13-1.05$ & 2318 \\
\hline POSentropy & $2.08 \mathrm{M}$ & $17.8-44.3-37.9$ & $1.16-1.11$ & 2282 & $2.11 \mathrm{M}$ & $14.2-41.5-44.3$ & $1.13-1.06$ & 2194 \\
\hline $\operatorname{trgNULL}$ & $2.35 \mathrm{M}$ & $23.2-45.1-31.7$ & $1.25-1.19$ & 0 & $2.42 \mathrm{M}$ & $19.9-44.1-36.0$ & $1.26-1.22$ & 0 \\
\hline $\operatorname{trgNULLpos}$ & $2.18 \mathrm{M}$ & $19.0-44.5-36.5$ & $1.18-1.14$ & 1625 & $2.16 \mathrm{M}$ & $14.7-41.6-43.7$ & $1.14-1.08$ & 1977 \\
\hline
\end{tabular}

Table 4: Tuple vocabulary and Ngram translation statistics for each segmentation strategy.

\begin{tabular}{|c|c|c|c|c|}
\hline \multicolumn{2}{|c|}{} & BLEU & mWER & NIST \\
\hline \hline \multirow{2}{*}{$\mathrm{E} \rightarrow \mathrm{S}$} & IBM1weight & 0.4714 & 40.22 & 9.83 \\
& POSentropy & $\mathbf{0 . 4 7 4 4}$ & 40.56 & $\mathbf{9 . 8 5}$ \\
\hline \hline \multirow{2}{*}{$\mathrm{S} \rightarrow \mathrm{E}$} & IBM1weight & 0.5470 & 34.41 & 10.74 \\
& POSentropy & 0.5466 & 34.44 & 10.72 \\
\hline \hline \multirow{2}{*}{$\mathrm{A} \rightarrow \mathrm{E}$} & alwaysNEXT & 0.3974 & 40.16 & 7.23 \\
& POSentropy & $\mathbf{0 . 4 0 2 4}$ & $\mathbf{4 0 . 0 5}$ & $\mathbf{7 . 3 9}$ \\
\hline
\end{tabular}

Table 5: Results with additional features.

In the large-vocabulary Spa-Eng tasks the target language and lexicon models add robustness to the whole system by penalising tuples with 'wrong' segmentations, or at least their catenation to build up the translation output. Yet the proposed segmentation achieves slightly better results in the Eng $\rightarrow$ Spa direction.

However, small-vocabulary tasks seem more sensitive to segmentation even when combining the core translation model with additional features, as improvements are more significant and higher than using only the translation model.

\section{Conclusion}

This paper delves into the details of tuple segmentation, a necessary step when training Ngram-based SMT systems. Apart from comparing all previously presented segmentation criteria, a novel strategy based on the entropy of the POS distribution is proposed.

A first conclusion of our study is that translation model performance is significantly affected by tuple segmentation, and the impact of segmentation strategies depends on language pair and corpus used, growing according to the percentage of tuples with NULL in source side. Secondly, the proposed linguistic criterion performs significantly better than segmentation strategies already presented in literature, behaving consistently across different translation tasks.

Regarding NULLs in target side, we conclude that they provide context to the model and removing them through resegmentation is not beneficial. Finally, when translation model is loglinearly combined with other features, direct impact of segmentation is smaller for large-vocabulary tasks, as features can partially compensate a bad tuple segmentation.

Possibly, the main drawback of the proposed segmentation strategy is that it requires a POS tagging tool. In this direction, one can think of making it independent from this by using induced classification strategies. Opposite to that, if chunking tools are available (for example in English), we would like to investigate ways of using chunking information to better segment tuples.

Another interesting issue is related to NULLs in target side. Whereas the presented experiments partially or fully removing
NULLs in target did not yield improvements, it appears that the best performing strategy is the one that 'uses' less units to NULL in translation. A further study of the reasons behind this is to be carried out, trying to understand the real contribution of this units and how or whether further improvements could be achieved by removing some of them.

Finally, other issues related to the tuple ngram translation model are pruning strategies and smoothing techniques. Though out of the scope of this paper, they are also crucial to understand the core model of ngram-based SMT. Therefore, a thorough study of their respective impact in translation quality is to be carried out.

\section{Acknowledgements}

This work was partly funded by European Union (TC-STAR project, FP6-506738), Generalitat de Catalunya and European Social Fund.

\section{References}

[1] P. Koehn and C. Monz, "Shared task: Statistical Machine Translation between European Languages," Proc. of the ACL Workshop on Building and Using Parallel Texts (ACL'05), pp. 119-124, 2005.

[2] M. Eck and C. Hori, "Overview of the IWSLT 2005 Evaluation Campaign," Proc. of the Int. Workshop on Spoken Language Translation, IWSLT'05, pp. 11-32, 2005.

[3] J. Mariño, R. Banchs, J. Crego, A. de Gispert, P. Lambert, M. R. Costa-jussà, and J. Fonollosa, "Bilingual N-gram statistical machine translation," Proc. of the MT Summit X, pp. 275-282, 2005.

[4] E. Vidal, "Finite-state speech-to-speech translation," Proc. of 1997 IEEE Int. Conf. on Acoustics, Speech and Signal Processing, pp. 111-114, 1997.

[5] J. M. Crego, J. Mariño, and A. de Gispert, "Finite-state-based and phrase-based statistical machine translation," Proc. of the 8th Int. Conf. on Spoken Language Processing, ICSLP'04, pp. 37-40, 2004.

[6] J. M. Crego, J. Mariño, and A. Gispert, "TALP: The UPC tuple-based SMT system," Proc. of the Int. Workshop on Spoken Language Translation, IWSLT'05, pp. 191-198, 2005.

[7] A. de Gispert and J. Mariño, "TALP: Xgram-based Spoken Language Translation System," Proc. of the Int. Workshop on Spoken Language Translation, IWSLT'04, pp. 85-90, 2004.

[8] P. Brown, S. Della Pietra, V. Della Pietra, and R. Mercer, "The mathematics of statistical machine translation," Сотриtational Linguistics, vol. 19, no. 2, pp. 263-311, 1993. 